\title{
云南拉沙山黑白仰鼻猴交配行为和出生季节
}

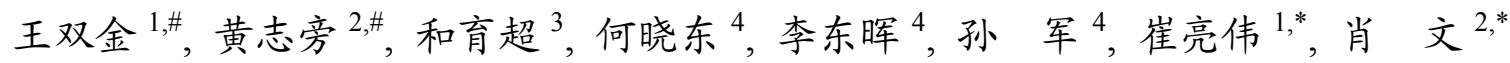

(1. 西南林业大学 云南省森林灾害预警与控制重点实验室, 云南 昆明 650224; 2. 大理学院 东喜玛拉雅资源与环境研究所, 云南 大理 671003 ;

3. 云南省怒江州兰坪县云岭省级自然保护区, 云南 兰坪 671400; 4. 高黎贡山国家级自然保护区 怒江管理局, 云南 六库 673100)

摘要: 灵长类的交配模式对于了解和掌握雄性的交配策略和社群的稳定机制非常重要, 但是目前有关亚洲灵 长类交配模式的数据较少; 因此, 该研究于 2011 年 1-12 月, 分别采用全事件取样法和焦点动物-瞬时扫描取样 法收集了拉沙山黑白仰鼻猴群的交配行为和出生数据。猴群全年交配, 有 2 个峰期, 一个是繁殖交配高峰期 (8一 10 月); 另一个在出生季节, 但其非繁殖交配的生物学意义尚不清楚。雌性通过俯卧/注视雄性或跳落邀配。爬跨 射精比为 8.8 , 射精交配稀少 (11.4\%), 这说明雄性并非每次交配都射精, 因而支持黑白仰鼻猴交配模式的主体为 多次爬跨射精或处于从单次爬跨射精到多次爬跨射精连续谱上段的观点。雄性邀配的射精爬跨多于雌性, 说明多 次爬跨射精不仅是雄性的一个交配策略, 而且可以决定交配模式在连续谱的位置。交配时间后延 6 7 个, 交配 频次与贞猴出生率相关。拉沙山猴群出生模式为严格的季节性, 这进一步证实了前人的观点。婴猴出生具有一定 的同步性，且不同猴群贞猴出生的同步模式不同。

中图分类号: Q959.848;Q492.6 文献标志码: A 文章编号：0254-5853-(2012)03-0241-08

\section{Mating behavior and birth seasonality of black-and-white snub-nosed monkeys (Rhinopithecus bieti) at Mt. Lasha}

\author{
WANG Shuang-Jin ${ }^{1, \#}$, HUANG Zhi-Pang ${ }^{2, \#}$, HE Yu-Chao ${ }^{2}$, HE Xiao-Dong ${ }^{3}$, LI Dong-Hui ${ }^{3}$, \\ SUN Jun ${ }^{3}$, CUI Liang-Wei ${ }^{1, *}$, XIAO Wen ${ }^{4, *}$ \\ (1. Key Laboratory of Forest Disaster Warning and Control in Yunnan Province, Southwest Forestry University, Kunming, Yunnan 650224, China \\ 2. Institute of Eastern-Himalaya Biodiversity Research, Dali University, Dali, Yunnan 671003, China; 3. Yunling Provincial Nature Reserve, Lanping, \\ Yunnan 671400, China; 4. Nujiang Administration Bureau, Gaolinggongshan National Nature Reserve, Liuku, Yunnan 673100, China)
}

\begin{abstract}
Copulation patterns are important to understanding male mating strategies and stabilization strategies of social organizations in primates. However, information on copulation patterns of Asian primates is relatively rare. This study was undertaken to collect data on mating behavior and birth seasonality of Black-and-white Snub-nosed monkeys (Rhinopithecus bieti) using all occurrence sampling and Focal animal-scan sampling methods at Mt. Lasha, between January and December, 2011. Our study focused on observing mating frequency and birth rates. Snub-nosed monkeys mate year round, with two observable peaks: one reproductive peak during the mating season, roughly from August to October, and a second non-breeding peak during the birth season. It is unclear what biological significance this non-reproductive mating peak has. During our observation, we noted a lower ratio of mount to ejaculation and rare ejaculatory copulations, which indicated that every mating would not result in ejaculation. This study corroborates the previous view that the Rhinopithecus bieti's copulatory pattern is likely multiple-mount ejaculation (MME) or at the upper part of mating continuum of single-mount ejaculation (SME) toward MME. More ejaculatory copulations initiated by males than females indicate that MME is not only a mating strategy of males, but that males can influence the position of their copulatory pattern on the continuum between SME and MME. The mating frequencies significantly correlated
\end{abstract}

收稿日期: 2012-02-03; 接受日期: 2012-03-27

基金项目: 国家自然科学基金 (31160422, 30960084, 30960085); 云南省森林灾害预警与控制重点实验室 (ZK09A313); 云南省重点学科野生动 植物保护与利用 (XKZ200904); 大理学院青年教师科研基金项目 (KYQN201123)

*通信作者(Corresponding authors), E-mail: Email: cuilw@yahoo.com; xiaowen.dali@gmail.com

\#共同第一作者(Authors contributed equally to the work)

第一作者简介: 王双金(1986一), 男, 硕士, 研究方向为灵长类行为生态学。E-mail: wsj2144584@163.com 黄志旁(1982-), 男, 硕士, 研究方向为灵长类营养生态学、行为生态学和保护生物学。E-mail: huangzp1982@gmail.com 
with the birth rates with a delay of 6 to 7 months. Monkeys gave birth within a strict seasonality with a birth peak of March, which confirms the previous view. Infants were born with a certain degree of synchronization, but different populations displayed different modes of synchronizations.

Key words: Rhinopithecus bieti; Copulatory pattern; Mating behavior; Birth seasonality

黑白仰鼻猴 (Rhinopithecus bieti，俗名滇金丝 猴) 隶属于灵长目猴科 (Cercopithecidae) 疮猴亚科 (Colobinae)仰鼻猴属 (Rhinopithecus)，是中国 I 级、 特有珍稀濒危灵长类，也是世界上分布海拔最高的 非人灵长类之一。该物种仅分布于金沙江和澜沧江 间的狭窄区域 (E98 $\left.37^{\prime} \sim 99^{\circ} 41^{\prime} ; \mathrm{N} 26^{\circ} 14^{\prime} \sim 29^{\circ} 20^{\prime}\right)$, 由南到北依次隶属于行政区划上的云南省云龙、兰 坪、丽汇、维西、德钦及西藏芒康等 6 个县 (Long et a1, 1994)。现存约 13 群，1500 1700 只 (Xiao et al, 2003)。猴群栖息地海拔由北到 南逐渐降低, 生境类型由以针叶树为主的暗针叶林 渐变为针阔混交林, 其主食由松萝 (Kirkpatrick et al, 1998) 渐变为阔叶 (Ding \& Zhao, 2004) 和竹叶 (Yang \& Zhao, 2001)。黑白仰鼻猴的社会由多个一 雄多雌单元 (one-male multiple- female unit: OMU) 和全雄单元 (all-male unit: AMU) 构成 (Cui et al, 2008; Kirkpatrick \& Grueter, 2010; Kirkpatrick et al, 1998, Huang et al, 2012)。

灵长类交配模式二分为单次爬跨射精 (single mount-to-ejaculation: SME) (Caldecott, 1986; Macaca spp: Fooden, 1980; N. larvatus: Yeager, 1990) 和多 次爬跨射精 (multiple mount-to-ejaculation: MME) (C. badius 和 C. guereza: Struhsaker, 1975) 或两者 兼有 (Caldecott, 1986; Macaca spp: Fooden, 1980; P. entellus: Hrdy, 1977; R. roxellana: Ren et al, 1995)。 笼养黑白仰鼻猴交配模式的主体为 MME 或处于从 SME 到 MME 交配模式连续谱的上段 (Cui et al, 2006)。尽管基于野生猴群的部分数据推测其交配模 式为 SME ( $n=11$, Kirkpatrick et al, 1998), 或是 SME 和 MME 并存 ( $n=8$, Xiang \& Sayers, 2009), 但是缺 乏定量系统数据的支持。到目前为止, 疮猴亚科其 他物种也未有交配模式的相关报道 (Asian colobines: Borries \& Koenig, 2008; R. bieti: Huang et al, 2012; Trachypithecus leucocephalus: Jin et al, 2009; Qi et al, 2008; $R$. roxellana: Ren et al, 2002, 2003; R. brelichi: Yang et al, 2009)。黑白仰鼻猴交配 模式信息仅源于笼养单个成年雄性, 缺乏代表性。
此外, 由于观察条件的限制 (如观察距离远、林密、 雨/雾大和猴群怕人等), 野生猴群繁殖特性的报道 多基于零星的观察记录。经过系统考察发现拉沙山 猴群是该物种目前较为理想的野生研究种群。通过 对其的系统跟踪观察，可以阐明黑白仰鼻猴交配模 式, 有助于理解其交配策略。

拉沙山黑白仰鼻猴的出生期为 2 月 15-4 月 7 日，且年间有变化 (如 2009 年出生时期为 2 月 21 -4 月 2 日, 2010 年为 2 月 15-4 月 7 日) ( Huang et $\mathrm{al}, 2012)$ 。动物的繁殖受多种因素的影响和调控, 短 期数据难以展示年际变化, 说明其繁殖季节性, 因 此, 本研究将进一步验证拉沙山黑白仰鼻猴群的繁 殖季节性。

\section{1 研究地点和方法}

\section{1 地点}

研究地位于云南省怒江州兰坪县云岭省级自 然保护区拉沙山 $\left(\mathrm{N} 26^{\circ} 20^{\prime}, \mathrm{E} 99^{\circ} 15^{\prime}\right)$, 行政区划隶 属兰坪县啦井镇期井村。拉沙山最高峰海拔 3854 $\mathrm{m}$ 。目前, 拉沙山猴群已呈孤立状态：其家域被低 海拔 $(<2800 \mathrm{~m})$ 村庄和耕地以及 $>3600 \mathrm{~m}$ 的高山 牧场包围，两者将猴群包围在海拔 2900 $3600 \mathrm{~m}$ 的林带内。猴群活动区植被随海拔从低到 高呈现带状分布，依次为针叶林、针阔混交林、落 叶阔叶林和暗针叶林 (Huang, 2009)。拉沙山地区最 低月均温 $4.4{ }^{\circ} \mathrm{C}\left(2\right.$ 月), 最高月均温 $17.5{ }^{\circ} \mathrm{C}$ (7 月)。 5 - 10 月为雨季, 降水量, 占全年的 79\%; 11 月至 翌年 4 月为干季 (Huang, 2009)。

研究对象为分布于黑白仰鼻猴分布区次南部 (与最南端的龙马山猴群直线距离约 $10 \mathrm{~km}$ ) 的拉沙 山猴群。猴群由 11 个 $\mathrm{OMU}$ 和 1 个 $\mathrm{AMU}$ 组成, 种 群大小约 130 只。猴群家域面积约 $11 \mathrm{~km}^{2}$ 。猴群生 境斑块化明显, 家域内零星有牧场、火烧地和烧炭 地 (砍伐栋树烧炭)。

\section{2 方法}

2011 年 1 一 12 月 (除 1 月雪大和 6 月雾大无 法跟踪猴群外), 在猴群活动区对面的突出地点 (如 
山脊和岩石), 借助单筒望远镜 (Nickon Fieldscope ED 60) 观察猴群, 当发现交配行为时, 采用全事件 取样法 (all-occurrence) (Altmann, 1974) 记录交配 数据 (包括发生时间、邀配者和接收者、邀配方式、 交配持续时间、骨盆推进次数、有无干扰以及干扰 者的年龄-性别以及交配后行为等), 累计观察时间 $927 \mathrm{~h}$ 。

交配行为包括邀配、爬跨、插入、骨盆推进、 射精和交配结束 (Cui et al, 2006; Xiang \& Sayers, 2009)。如果雄猴爬跨雌猴, 伴有骨盆推进, 则记为 交配; 若只爬跨, 无骨盆推进, 不记为交配。骨盆推 进停止后保持最大插入, 且有明显停顿 ( $\geqslant 3 \mathrm{~s})$ 视 为射精 (Cui et al, 2006)。

2011 年 2 月 16 日-4 月 12 日, 在猴群活动区 对面的突出地点借助单筒望远镜采用焦点动物 - 瞬 时扫描取样法记录猴群受食或集体移动过程中 (如 过沟和视野开阔地等) 婴猴 (1.5 月内均由母亲携 带) 和成年雌猴数量, 统计婴猴和成雌数比值 $(\mathrm{I} / \mathrm{F}$ : number of infants: number of adult females) (Altmann, 1974)。猴群包括 29 只成年雌性, 为避免成年雌性 数量波动导致研究结果偏差, 当成䧳数 $>15$ 时, 记 录的 I/F 值才纳入统计 (Huang et al, 2012)。借助 I/F 值和婴猴数的变化, 可阐明猴群的出生模式 (Xiang \& Sayers, 2009; Huang et al, 2012)。

\section{3 数据分析}

采用 Proportion test 检验雌雄两性邀配、雌性邀 配方式以及雌雄两性邀配的射精爬跨间的差异。采 用 $t$-test 检验交配参数差异。采用 goodness- of-fit test 检验交配季节性。通过 Circular statistics 分析出生 季节性, 并采用 Rayleigh test $\left(Z=n r^{2}\right)$ 检验出生模式 (Batschelet, 1981; Zar, 1999)。中位出生日期、平均 出生日期及标准差的计算参照 Eisenberg et al (1981) 的方法。

\section{2 结 果}

\section{1 交配频次}

除 1 月 (雪大) 和 6 月 (雾大) 观察条件限制无 法搜集数据外，其他月份均观察到交配行为。2011 年共记录到 57 次交配，其中完整交配 (从交配开始 到结束) 44 次, 不完整交配 (漏掉交配的某个/些环 节) 13 次 (表 1)。

\section{2 邀配}

雌性有两种邀配方式: (1) 俯卧并注视雄性
(PG: prostration and repeatedly glancing at the male), 即䧳性跑到雄性面前，腹部紧贴树干，四肢完全弯 曲、低头、慰部微微尧起, 不时 (扭头) 注视雄性片 刻; (2) 跳落 (jumping: JP), 即雌性突然从高处跳/ 跑到雄性面前, 然后蹲伏或俯卧。

表 1 拉沙山黑白仰鼻猴观察时间和交配频次

Tab. 1 Mating duration and frequency of Rhinopithecus bieti at Mt. Lasha

\begin{tabular}{cccccccccccccc}
\hline $\begin{array}{c}\text { 月份 } \\
\text { Month }\end{array}$ & 1 & 2 & 3 & 4 & 5 & 6 & 7 & 8 & 9 & 10 & 11 & 12 & $\begin{array}{c}\text { 总和 } \\
\text { Total }\end{array}$ \\
\hline $\begin{array}{c}\text { 时间 } \\
\text { Duration (h) }\end{array}$ & 0 & 71.5 & 77 & 89.5 & 78.5 & 0 & 80.5 & 83.5 & 110 & 72 & 145 & 121 & 927.5 \\
$\begin{array}{c}\text { 交配次数 } \\
\text { Mating } \\
\text { frequency }\end{array}$ & 0 & 1 & 3 & 4 & 3 & 0 & 2 & 8 & 15 & 12 & 7 & 2 & 57 \\
$\begin{array}{c}\text { 完整交配 } \\
\text { Complete } \\
\text { mating }\end{array}$ & 0 & 1 & 1 & 3 & 3 & 0 & 2 & 4 & 11 & 10 & 7 & 2 & 44 \\
$\begin{array}{c}\text { 不完整交配 } \\
\text { Incomplete } \\
\text { mating }\end{array}$ & 0 & 0 & 2 & 1 & 0 & 0 & 0 & 4 & 4 & 2 & 0 & 0 & 13 \\
\hline
\end{tabular}

44 次完整交配记录中, 邀配 36 次, 雄猴直接爬 跨 8 次。雌性邀配多于雌性 ( $75 \%$ vs $25 \%$, Proportion test: $Z=3.46, P<0.001)$ 。雌性邀配 27 次, 其中 $P G$ 占 $59 \%(n=16), \mathrm{JP}$ 占 41\% $(n=9)$, 雌性邀配方式无差异 (Proportion test: $Z=0.98, P=0.16$ )。

\section{3 直接爬跨}

在无冲突发生和邀配行为的情况下，雄性直接 走到雌性身后抓抬雌性臀部随即爬跨, 并有骨盆推 进。雄性直接爬跨 8 次, 占完整交配记录的 $18 \%$ $(n=44)$ 。

\section{4 交配}

交配姿势为腹一背式: 雄性从雌性后面爬跨, 两 前肢抱住雌性胯部或后腰进行骨盆推进。保持俯卧 状态的雌性摆动头部或以臀部为中心旋转身体 上部。

交配对象可识别的 57 次交配中, 雄猴与成年 雌性交配 54 次，与青年雌性交配 3 次。射精与否可 辨别的 44 次记录中，5 次射精记录分布于 7-10 月 (7 和 9 月各 1 次, 8 和 10 月各 2 次), 占 $11.4 \%$ 。爬 跨射精比为 8.8 。

雌雄邀配的爬跨时间和骨盆推进次数无差异, 但雄性邀配的射精交配多于雌性 $(23.5 \%$ vs 3.7, $n_{1}=17, n_{2}=27$, Proportion test: $Z=2.02, P=0.022$ )。射精 爬跨骨盆推进次数和持续期多/长于非射精爬跨骨 盆推进次数和持续期, 但不显著 (表 2)。 
表 2 拉沙山黑白仰鼻猴交配参数

Tab. 2 Copulatory parameters of male Rhinopithecus bieti at Mt. Lasha

\begin{tabular}{|c|c|c|c|}
\hline $\begin{array}{c}\text { 变量 } \\
\text { Variable }\end{array}$ & $\begin{array}{c}\text { 平均值 } \pm \text { 标准差 (范围) } \\
\text { mean } \pm S D \text { (range) }\end{array}$ & $n$ & $\begin{array}{c}\text { 差异性检验 } \\
\text { Test of difference }\end{array}$ \\
\hline $\begin{array}{l}\text { 爬跨时间 } \\
\text { Duration of mounts }\end{array}$ & $12.7 \pm 3.1(5-16)$ & 44 & \multirow{4}{*}{$t_{42}=1.48, P=0.15$} \\
\hline $\begin{array}{l}\text { 骨盆推进次数 } \\
\text { Number of thrusts }\end{array}$ & $9.8 \pm 2.6(4-13)$ & 44 & \\
\hline $\begin{array}{l}\text { 雌性邀配的交配爬跨时间 (s) } \\
\text { Duration of mounts initiated by females }\end{array}$ & $13.2 \pm 2.9(5-16)$ & 27 & \\
\hline $\begin{array}{l}\text { 雄性邀配的交配爬跨时间(s) } \\
\text { Duration of mounts initiated by males }\end{array}$ & $11.8 \pm 3.2(7-16)$ & 17 & \\
\hline $\begin{array}{l}\text { 雌性邀配的交配骨盆推进次数 } \\
\text { Thrusts in mounts initiated by females }\end{array}$ & $10.1 \pm 2.5(4-13)$ & 27 & \multirow[b]{2}{*}{$t_{42}=1.15, P=0.25$} \\
\hline $\begin{array}{l}\text { 雄性邀配的交配骨盆推进次数 } \\
\text { Thrusts in mounts initiated by males }\end{array}$ & $9.2 \pm 0.8(5-13)$ & 17 & \\
\hline $\begin{array}{l}\text { 射精爬跨骨盆推进次数 } \\
\text { Thrusts in mounts with ejaculation }\end{array}$ & $11.8 \pm 0.8(11-13)$ & 5 & \multirow[b]{2}{*}{$t_{42}=1.91, P=0.063$} \\
\hline $\begin{array}{l}\text { 非射精爬跨骨盆推进次数 } \\
\text { Thrusts in mounts without ejaculation }\end{array}$ & $9.5 \pm 2.6(4-13)$ & 39 & \\
\hline $\begin{array}{l}\text { 射精爬跨持续期 (s) } \\
\text { Duration of mounts with ejaculation }\end{array}$ & $15.0 \pm 0.7(14-16)$ & 5 & \multirow{2}{*}{$t_{42}=1.82, P=0.075$} \\
\hline $\begin{array}{l}\text { 非射精爬跨持续期 (s) } \\
\text { Duration of mounts without ejaculation }\end{array}$ & $12.4 \pm 3.2(5-16)$ & 39 & \\
\hline
\end{tabular}

$\bar{t}$ 值相邻两个交配参数间的差异采用 $t$-test 检验(Differences between two copulation parameters bordering upon $t$ values were tested by $t$-test)。

\section{5 性打摚}

共记录到 4 次性打摚, 3 月和 4 月各 2 次。干扰 个体均为 1 岁幼猴。曾观察到在交配过程中, 1 2 只幼猴会在交配者周边 $1 \mathrm{~m}$ 内跳跃，不时伸前肢抓 雌性。但幼猴的干扰并未中断雌雄交配。交配后，雄 猴静坐，雌猴离去，其中小猴紧跟母亲后吃奶 1 次, 被拒 2 次; 小猴紧随母亲离去 1 次。

\section{6 交配后行为}

交配后梳理 11 次，占 $19.3 \%(n=57)$ : 其中，雌 性梳理雄性 8 次, 占 $73 \%(n=11)$; 雄性梳理雌性 1 次, 占 $9 \%$; 雄猴离去, 雌猴自我梳理 1 次; 雄性静 坐, 雌性在其 $1 \mathrm{~m}$ 内自我梳理 1 次。雄性原地静坐, 雌性离开 35 次 (61.4\%); 雄性原地静坐, 雌性在雄 性 $1 \mathrm{~m}$ 内取食 7 次 $(13.2 \%)$; 两者原地休息 1 次, 两者同时原地取食 1 次; 雌猴静坐, 雄猴离开 2 次。

\section{7 交配季节}

全年记录到 57 次交配。2-7 月交配频次低, 8 月逐渐增加, 10 月达到峰值, 随后逐渐降低。尽管 猴群全年交配, 但并非均匀分布 $\left(\chi^{2}=35.11\right.$, goodness-of-fit test, $d f=9, P<0.001)$; 其峰期为 $8-10$ 月 (图 1)。

\section{8 出生季节}

2011 年 2 月 19 日首个㚣猴出生, 从 2 月 19 日 - 2 月 25 日, $\mathrm{I} / \mathrm{F}$ 斜率逐渐增加说明贞猴出生渐多; 2 月 25 日一 3 月 12 日, 斜率变缓, 表明新增婴猴数减
缓； 3 月 12 日一 18 日，斜率增大，说明肾猴出生增 多; 3 月 18 日一 4 月 12 日，尽管 $\mathrm{I} / \mathrm{F}$ 波动，但婴猴继 续出生，且婴猴数在 4 月 12 日达到最大值 (13 只)。 随后 $\mathrm{I} / \mathrm{F}$ 和㚣猴数虽波动, 但贞猴数一直稳定在 13 只, 表明 4 月 12 日出生结束 (图 2), 因此, 2011 年 猴群新生贞猴 13 只，出生期为 2 月 19 日一 4 月 12 日, 出生高峰为 3 月 (图 1)。

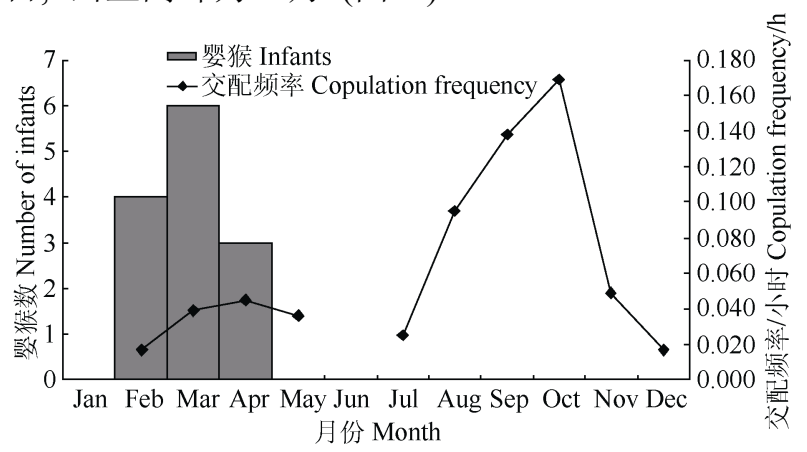

图 1 拉沙山黑白仰鼻猴婴猴数和交配频率分布

Fig. 1 Distribution of number of infants and mating frequencies of Rhinopithecus bieti at Mt. Lasha

1 月雪大、 6 月雾导致无法跟踪猴群，这两个月的数据缺失。

It is impossible to track the monkeys in January due to snow and in June due to fog . No data exists for these two months.

拉沙山猴群出生模式为严格的季节性 $(r=0.96$, $Z=12.01, P<0.001)$ 。猴群平均出生期 3 月 22 日 $(S D=16 \mathrm{~d})$, 中位出生期 3 月 19 日。

拉沙山猴群第 1 周 $(2$ 月 19 - 25 日)、第 4 和 7 周贞猴出生较多 (占 $62 \%$ ), 其他时间新生㚣猴较 


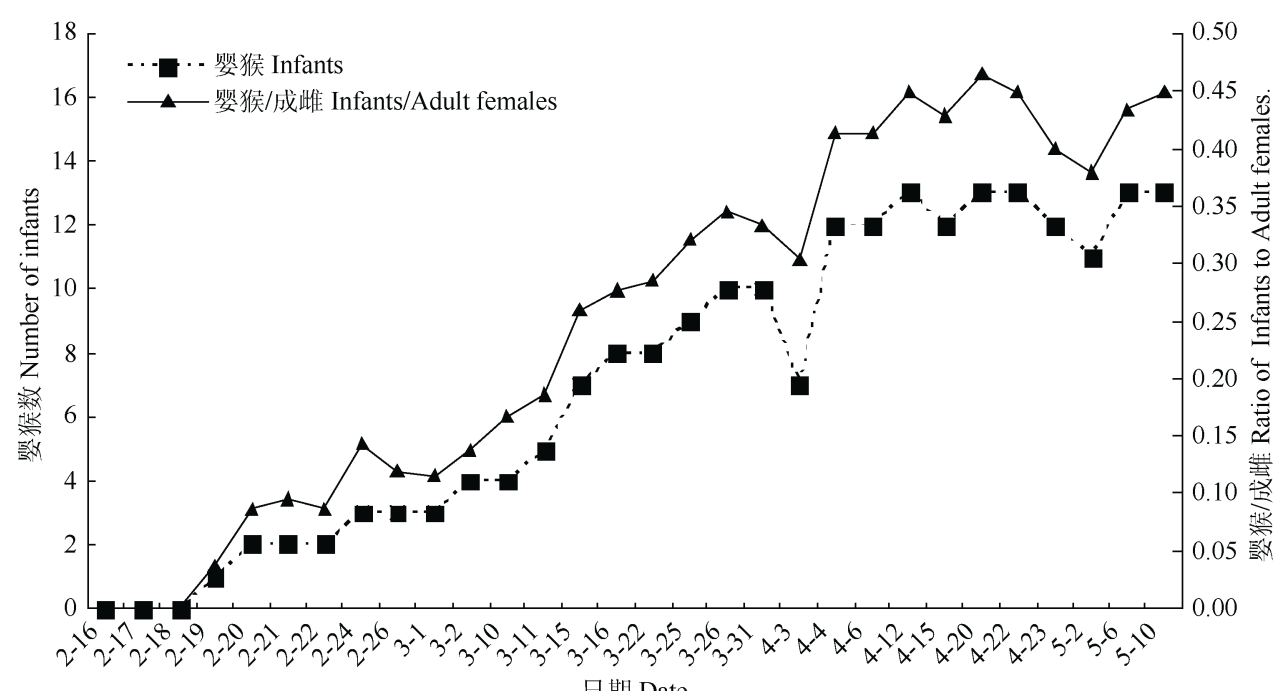

图 2 拉沙山黑白仰鼻猴贞猴/成䧳比 $(\mathrm{I} / \mathrm{F})$ 和紧猴数变化

Fig. 2 Change in number of infants and ratio of Infants to Adult Females (I/F) of Rhinopithecus bieti at Mt. Lasha

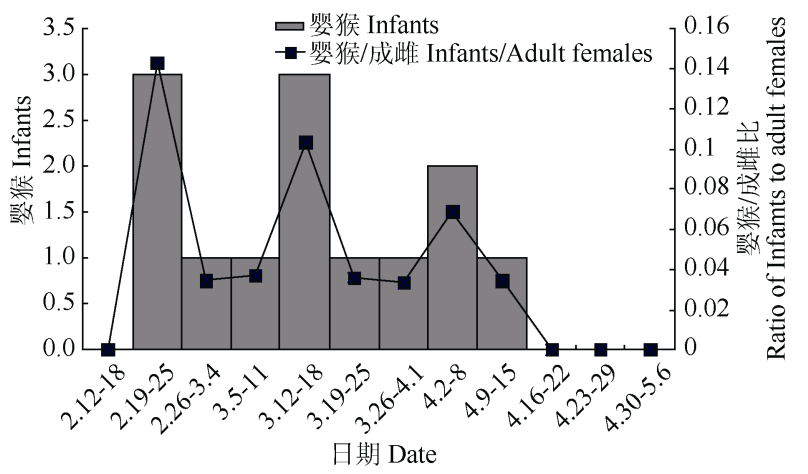

图 3 拉沙山黑白仰鼻猴每周婴猴/成雌比和新生婴猴数

Fig. 3 Number of infants and weekly variation in the ratio of infants to adult females (I/F) of Rhinopithecus bieti at Mt. Lasha

少, 第 9 周出生结束, 因此, 贞猴出生具有一定的 同步性 (图 3)。

\section{9 交配和出生的相互关系(时间后移)}

交配时间后延 6 7 个月, 交配频次和婴猴出生 率相关 (后延 6 个月: $R^{2}=0.68, F_{1,8}=17.27, P=0.0032$; 后延 7 个月: $\left.R^{2}=0.76, F_{1,8}=25.07, P=0.001\right)$ 。

\section{3 讨 论}

\section{1 交配季节}

拉沙山黑白仰鼻猴全年交配, 有 2 个高峰期, 最高峰在交配季节; 另一峰期在出生季节。这进一 步证实了以前的报道 (Huang et al, 2012)。根据拉沙 山猴群射精交配仅出现在交配季节 (7-10 月)、婴 猴出生峰期 (2-4 月) 及其 $195 \mathrm{~d}$ 的怀孕期 (Ji et al, 1998), 可以确定猴群繁殖交配峰期为 8-10 月, 这
与仰鼻猴属其他物种的报道一致 (R. bieti: Cui et al, 2006; Kirkpatrick et al, 1998; Xiang \& Sayers, 2009; $R$. roxellana: Zhang et al, 2000; Qi et al, 2008; $R$. brelichi: Yang et al, 2009), 但繁殖交配期外, 非受孕 交配的生物学意义有待深入研究。

\section{2 邀配}

笼养黑白仰鼻猴䧳性有 3 种邀配方式，分别为 俯卧并注视雄性、跳落和上下摆头 (Cui et al, 2006); 而在野生猴群中仅观察到前 2 种邀配方式 (Xiang \& Sayers, 2009)。黑白仰鼻猴雌性以上下摆头的邀 配方式 (Cui et al, 2006; Xiang \& Sayers, 2009) 在 其他物种中有报道 (Nasaslis larvatus: Yeager, 1990)。野生与笼养黑白仰鼻猴䧳性邀配方式的差异 可能与观察条件 (如林密和距离远等) 以及猴群/ 个体不同有关。总之，黑白仰鼻猴雌性邀配方式与 疮猴亚科其他物种典型的邀配方式基本一致 (Pygathrix nemaeus: Kavanagh, 1978; Lippold, 1998; Presbytis entellus: Newton, 1987; R. roxellana: Ren et al, 1995, 2002; Colobus badius: Struhsaker, 1975; N. larvatus: Yeager, 1990)。

笼养雄性以伴有特别叫声、露齿动领表情邀配 (Cui et al, 2006), 这在疮猴亚科其他物种中亦有报 道 (P. nemaeus: Kavanagh, 1978; Lippold, 1998; R. roxellana: Qi, 1988; Ren et al, 1995)。野生黑白仰鼻 猴雄性以突然转向雌猴、瞪眼方式邀配 (Xiang \& Sayers, 2009)。遗憾的是, 本研究未能准确、全面的 观察到雄性邀配; 因此, 也无法确认雄性的邀配 方式。 
交配中，雌性保持匍司状态或以臀部为中心作 前身的弧形移动, 或仅左右摆动头部并有时停下回 头注视雄性, 或保持不动而注视前方 (Cui et al, 2006)。本研究观察到前 2 种䧳性行为, 而没有观察 最后一种行为, 因此, 目前基本可以确定雌性的交 配行为, 但仍需进一步观察来确定是否还有其他的 交配行为, 是否具有个体/群体差异。

\section{3 交配模式}

笼养黑白仰鼻猴爬跨射精比为 5.2, 单次爬跨 射精约占 4.4\% (Cui et al, 2006)。拉沙山猴群爬跨射 精比为 8.8 , 射精交配稀少(11.4\%), 这意味着雄性 并非每次交配都射精。小昌都猴群也如此 (但缺乏 定量数据支持) (Xiang \& Sayers, 2009), 因此, 黑白 仰鼻猴交配模式的主体应为 MME 或处于从 SME 到 MME 连续谱上段 (Cui et al, 2006)。这与疮猴亚 科其他物种的报道一致 (Presbytis entellus: Hrdy, 1977; P. cristata, P. johnii, C. badius, C. guereza: Hrdy \& Whitten, 1987; R. roxellana: Ren et al,1995)。

除类人猿外的 53 种灵长类中, $50 \%$ 是 $\mathrm{SME}$, 43\% 是 MME (或 “Serial” ), 而 7\%的物种两者兼有 (Hrdy \& Whitten, 1987)。Macaca 属的交配模式是从 SME 到 MME 的连续谱, 而且, 交配模式与雄性间 的容忍度有关: SME 物种 (如 M. radiata, $M$. sylvanus, M. sinica, M. arctoides 等) 雄性间容忍度 高、竞争不激烈且缺乏严格的社会等级, MME 物种 (如 M. mulatta, M. fuscata, M. nigra 和 M. silenus 等) 雄性间容忍度低、竞争激烈且有社会等级 (Shively et al, 1982)。Caldecott (1986) 认为雄性在松弛的社 会环境中的交配模式是 SME, 在敌对环境中则为 MME。

根据性选择理论 (Darwin, 1871; Trivers, 1972), 拉沙山猴群成年性比 (1M: 3F) 和体重性二型 $(\mathrm{M} / \mathrm{F} \approx 2: 1$, Kirkpatrick, 1996) 暗示了以 $\mathrm{OMU}$ 为基本 繁殖单元的雄性间有较强的交配竞争。拉沙山猴群 雌、雄激配的交配爬跨时间和骨盆推进次数无差异, 但是雄性邀配的射精交配多于雌性, 这说明 MME 不仅是雄性的一个交配策略, 而且雄性可以决定交 配模式在连续谱上的位置。另外, 笼养黑白仰鼻猴 雌性邀配的射精爬跨多于非射精爬跨暗示了雌性 的激励对雄性的性觉醒是个积极因素, 这意味着雌 性的交配态度也会影响交配模式在连续谱的位置 (Cui et al, 2006; Zhao, 1993)。

拉沙山雄猴射精与非射精爬跨骨盆推进次数
和爬跨时间无差异; 但是, 笼养雄猴射精爬跨时间 和骨盆推进次数长/多于非射精爬跨时间和骨盆推 进次数 (Cui et al, 2006)。这可能与竞争性社会环境 压力大小不同有关: 野生猴群社会竞争压力大, 笼 养猴群社会竞争压力小。

\section{4 交配后行为}

交配后雌性梳理雄性多于雄性梳理雌性 $(73 \%$ vs 9\%), 且雌性邀配导致的交配多于雄性 (75\% vs $25 \%$, 因此, 雌性梳理雄性可能是对交配的一种回 报 (Manson, 2004)。

\section{5 出生季节}

疮猴亚科灵长类出生模式有 3 种: 严格的季节 性、出生高峰期和无规律性 (Struhsaker \& Leland, 1987)。绝大多数猴亚科灵长类出生具有严格的季节 性 (Andelman, 1986)。拉沙山猴群出生模式为严格 的季节性 (Huang et al, 2012), 且为脉冲型 ( $S D=16$ d $<30$ d: Caughley, 1977)。野生黑白仰鼻猴猴群出 生期为 2 月 15-4 月 12 日，峰期 3 月 (Huang et al, 2012; Kirkpatrick et al, 1998; Xiang \& Sayers, 2009)。 笼养黑白仰鼻猴出生于 12-6 月, 峰期 3-5 月, 平 均出生期 4 月 18 日 (标准差 $43 \mathrm{~d}$ ); 尽管出生分散 但具高峰期 (Cui et al, 2006)。黑白仰鼻猴群季节性 繁殖的触发因素 (或近因 Proximate cause) 与光周 期无关 (Cui et al, 2006; Huang et al, 2012), 而与气 候和食物资源供给的季节性变化有关 (Huang et al, 2012)。

黑白仰鼻猴分布区次南部拉沙山猴群出生时 间跨度 $53 \mathrm{~d}$, 平均出生期 3 月 22 日 $(S D=16 \mathrm{~d})$; 中 位出生期 3 月 19 日。而分布区次北端小昌都猴群 出生跨度 $39 \mathrm{~d}$, 平均出生期 2 月 28 日 $(S D=3.6 \mathrm{~d})$, 中位出生期 2 月 24 日 (Xiang \& Sayers, 2009), 因此, 黑白仰鼻猴的出生模式具有明显的趋异适应特征: 北部猴群出生时间跨度比南部猴群短 $13 \mathrm{~d}$, 提前 15 $\mathrm{d}$ 出生, 平均出生期早 $28 \mathrm{~d}$, 中位期提前 $23 \mathrm{~d}$, 出生 期标准差减少 $12.4 \mathrm{~d}$ 。黑白仰鼻猴南北部两个猴群 家域纬度相差 $3^{\circ}$, 猴群出生相应提前、时间跨度缩 短、出生期标准差减少约 2 周, 中位出生期提前约 3 周, 平均出生期提前 4 周。也就是说, 随家域维度 和海拔升高, 出生季节性越显著。长尾叶猴家域纬 度越高, 其出生季节性越明显 (Newton \& Dunbar, 1994)。峨嵋山藏狝猴 (M. thibetana) 猴群中位出生 期随猴群家域海拔升高而提前 (Zhao \& Deng, 1988)。黑白仰鼻猴群出生季节性随海拔升高而增强 
可能与气候和食物供给季节性变化显著增强有关, 而具体的影响因素及其调控机制有待后续研究。

拉沙山猴群每周出生贞猴数不同, 小昌都猴群 也如此 (Xiang \& Sayers, 2009), 因此, 不同猴群贞 猴出生的同步性模式不同: 拉沙山猴群第 1 和 4 周 出生婴猴最多, 第 7 周次之; 而小昌都猴群第 2 周 最多，依次为第 1 和 4 周 (Xiang \& Sayers，2009); 因此, 长期、系统的研究有助于阐明婴猴出生同步 性模式的调控因素及其机制。

目前, 通常借助贞猴/成雌数比值 $(\mathrm{I} / \mathrm{F})$ 来研究 难以进行个体识别灵长类种群的出生模式 (Xiang \& Sayers, 2009; Huang et al, 2012)。由于野外跟踪和 观察条件的限制, I/F 值波动较大, 有时造成结束期 和出生模式的偏差; 如果同时考虑贞猴数, 就可以 避免这种偏差, 得到真实的结果。在本研究中, 根 据 $\mathrm{I} / \mathrm{F}$ 比的最大值判定出生结束期在 4 月 20 日, 事 实上，婴猴最大值的首次出现日期 (4 月 12 日) 应

\section{参考文献:}

Altmann J. 1974. Observational study of behavior: sampling methods [J]. Behav, 49(3): 227-267.

Andelman SJ. 1986. Ecological and social determinants of cercopithecine mating patterns [M] // Rubenstein DI, Wrangham RW. Ecological Aspects of Social Evolution. Princeton: Princeton University of Press, 201-216.

Batschelet E. 1981. Circular Statistics in Biology [M]. New York: Academic Press.

Borries C, Koenig A. 2008. Reproductive and behavioral characteristics of aging in female Asian colobines [M] // Atsalis S, Margulis SW, Hof PR. Primate Reproductive Aging: Cross-Taxon Perspective on Reproduction. Basel: Karger, 80-102.

Caldecott JO. 1986. Mating patterns, societies and the ecogeography of macaques [J]. Anim Behav, 34(1): 208-220.

Caughley G. 1977. Analysis of Vertebrate Population [M]. New York: John Wiley and Sons.

Cui LW, Huo S, Zhong T, Xiang ZF, Xiao W, Quan RC. 2008. Social organization of black-and-white snub-nosed monkeys (Rhinopithecus bieti) at Deqin, China [J]. Am J Primatol, 70(2): 169-174.

Cui LW, Sheng AH, He SC, Xiao W. 2006. Birth seasonality and interbirth interval of captive Rhinopithecus bieti [J]. Am J Primatol, 68(5): 457-563.

Darwin C. 1871. The Descent of Man, and Selection in Relation to Sex [M]. London: John Murray, Reprinted in 1998.

Ding W, Zhao QK. 2004. Rhinopithecus bieti at Tacheng, Yunnan: diet and daytime activities [J]. Int J Primatol, 25(3): 583-598.

Eisenberg JF, Dittus WPJ, Fleming TH, Green K, Struhsaker T, Trorington RW, JR. 1981. Techniques for the Study of Primate Population Ecology [M]. Washington: National Academy Press.

Fooden J. 1980. Classification and distribution of living macaques (Macaca lacepede) [M] // Lindburg DG. The Macaques: Studies in Ecology, Behavior and Evolution. New York: Van Nostrand Reinhold, 1-9.

Hrdy SB. 1977. The Langurs of Abu: Female and Male Strategies of
该是猴群的出生结束期 (图 2)。

疮猴亚科中，严格季节性出生种群的出生间隔 接近 24 个月, 而出生具有高峰期和无固定规律种 群的出生间隔接近 15 个月 (Newton \& Dunbar, 1994)。2009 年，拉沙山猴群 OMUs 有 24 只成年雌 性，其中 11 个产下贞猴; 2010 年 OMUs 有 27 个成 年雌性，其中 16 个产下贞猴 (Huang et al, 2012); 这说明另有 3 只雌性成年并产下婴猴。2011 年, 拉 沙山猴群有 29 只成年雌猴, 出生贞猴 13 只。这意 味着有 2 只雌性成年并生育后代，因此，猴群的出 生间隔为 2 年。

致谢: 云南省怒江州兰坪云岭省级自然保护区 管理局的领导及工作人员给予了本研究大力支持; 野外工作得到了大山箐社区居民的帮助; 野外助手 苏庆生和张金福协助完成了野外工作，在此一并表 示衰心感谢!

Reproduction [M]. Cambridge: Harvard University Press.

Hrdy SB, Whitten PL. 1987. Patterning of sexual activity [M] // Smuts B, Cheney D, Seyfarth R, Wrangham R, Struhsaker T. Primate Societies. Chicago: University of Chicago Press, 370-384.

Huang ZP. 2009. Foraging Food Choice, Reproduction and Sleeping Site Selection of Black-and-white Snub-nosed Monkey (Rhinopithecus bieti) at the Southern Range [D]. Thesis, Southwest Forestry University, Kunming. [黄志旁. 2009. 黑白仰鼻猴 (Rhinopithecus bieti) 南部种 群的摄食选择、繁殖及过夜地选择 [D]. 硕士学位论文, 西南林学 院，昆明.]

Huang ZP, Cui LW, Scott MB, Wang SJ, Xiao W. 2012. Seasonality of reproduction of wild black-and-white snub-nosed monkeys (Rhinopithecus bieti) at Mt. Lasha, Yunnan, China [J]. Primates, doi: 10.1007/s10329-012-0305-7.

Ji WZ, Zou RJ, Shang EY, Zhou HW, Yang SC, Tian BP. 1998. Maintenance and breeding of Yunnan snub-nosed monkeys (Rhinopithecus [Rhinopithecus] bieti) in captivity [M] // Jablonski NG. The Natural History of the Doucas and Snub-nosed Monkeys. Singapore: World Scientific, 323-335.

Jin T, Wang DZ, Zhao Q, Yin LJ, Qin DG, Ran WZ, Pan WS. 2009. Social organization of white-headed langurs (Trachypithecus leucocephalus) in the Nongguan Karst Hills, Guangxi, China [J]. Am J Primatol, 71(3): 206-213.

Kavanagh M. 1978. The social behaviour of Doucs (Pygathrix nemaeus nemaeus) at San Diego Zoo [J]. Primates, 19(1): 101-114.

Kirkpatrick RC. 1996. Ecology and Behavior of the Yunnan Snub-nosed Langur Rhinopithecus bieti (Colobinae) [D]. Dissertation. University of California Davis.

Kirkpatrick RC, Grueter CC. 2010. Snub-nosed monkeys: multilevel societies across varied environments [J]. Evol Anthropol, 19(3): 98-113.

Kirkpatrick RC, Long YC, Zhong T, Xiao L. 1998. Social organization and range use in the Yunnan Snub-nosed Monkey Rhinopithecus bieti [J]. 
Int J Primatol, 19(1): 13-51.

Lippold LK. 1998. Natural history of Douc langurs[M] // Jablonski NG. The Natural History of the Doucs and Snub-nosed Monkeys. Singapore: World Scientific Publishing, 191-206.

Long YC, Kirkpatrick CR, Zhong T, Xiao L. 1994. Report on the distribution, population, and ecology of the Yunnan snub-nosed monkey (Rhinopithecus bieti) [J]. Primates, 35(2): 241-250.

Manson JPH, Navarrete CD, Silk JB, Perry S. 2004. Time-matched grooming in female primates? New analyses from two species [J]. Anim Behav, 67(3): 493-500.

Newton PN. 1987. The social organization of forest hanuman langurs (Presbytis entellus) [J]. Int J Primatol, 8(3): 199-232.

Newton PN, Dunbar RTM. 1994. Their ecology, behavior, and evolution [M] // Davies AG, Oates JF. Colobine Monkeys. Cambridge: Cambridge University Press, 311-346.

Qi JF. 1998. Observation studies on reproduction of golden monkey in captivity. I. Copulatory behavior [J]. Acta Theriol Sin, 8(3): 172-175. [戚静芬. 1988. 人工饲养下金丝猴繁殖的观察研究 I. 交配 行为. 兽类学报, 8(3): 172-175.]

Qi XG, Li BG, Ji WH. 2008. Reproductive parameters of wild female Rhinopithecus roxellana [J]. Am J Primatol, 70(4): 311-319.

Ren BP, Xia SZ, Li QF, Zhang SY, Liang B, Qiu JH. 2002. Male copulatory patterns in captive Sichuan snub-nosed monkeys (Rhinopithecus roxellana) [J]. Acta Zool Sin, 48(5): 577-584. [任宝平, 夏述忠, 李庆 芬, 张树义, 梁冰, 邱军华. 2002. 圈养雄性川金丝猴交配模式. 动 物学报, 48(5): 577-584.]

Ren BP, Zhang SY, Xia SZ, Li QF, Liang B, Lu MQ. 2003. Annual reproductive behavior of Rhinopithecus roxellana $[\mathrm{J}]$. Int J Primatol, 24(3): 575-589.

Ren RM, Yan KH, Su YJ, Qi HJ, Liang B, Bao WY, De Waal FBM. 1995. The reproductive behavior of golden monkeys in captivity (Rhinopithecus roxellana roxellana) [J]. Primates, 36(1): 135-143.
Shively C, Clarke S, King N, Schapiro S, Mitchell G. 1982. Patterns of sexual behavior in male macaques [J]. Am J Primatol, 2(4): 373-384.

Struhsaker TT. 1975. The Red Colobus Monkey [M]. Chicago: University of Chicago Press.

Struhsaker TT, Leland L. 1987. Colobines: Infanticide by adult male [M] // Smuts BB, Cheney DL, Seyfarth RM, Wrangham RW, Struhsaker TT. Primate Society. Chicago: University of Chicago Press, 83-97.

Trivers RL. 1972. Parental investment and sexual selection [M] // Campbell B. Sexual Selection and the Descent of Man. Chicago: Aldine, 136-139.

Xiang ZF, Sayers K. 2009. Seasonality of mating and birth in wild black-and-white snub-nosed monkeys (Rhinopithecus bieti) at Xiaochangdu, Tibet [J]. Primates, 50(1): 50-55.

Xiao W, Ding W, Cui LW, Zhou RL, Zhao QK. 2003. Habitat degradation of Rhinopithecus bieti in Yunnan, China [J]. Int J Primatol, 24(2): 389-398.

Yang MY, Sun DY, Zinner D, Roos C. 2009. Reproductive parameters in Guizhou Snub-nosed monkeys (Rhinopithecus brelichi) [J]. Am J Primatol, 71(3): 266-270.

Yang SJ, Zhao QK. 2001. Bamboo leaf-based diet of Rhinopithecus bieti at Lijiang, China [J]. Folia Primatol, 72(2): 92-95.

Yeager CP. 1990. Proboscis monkey (Nasalis larvatus) social organization: group structure [J]. Am J Primatol, 20(2): 95-106.

Zar JH. 1999. Biostatistical Analysis [M]. 4th ed.. Prentice Hall, Upper Saddle River.

Zhang SY, Liang B, Wang LX. 2000. Seasonality of matings and births in captive Sichuan golden monkeys (Rhinopithecus roxellana) [J]. Am J Primatol, 51(4): 265-269.

Zhao QK. 1993. Sexual behavior of Tibetan macaques at Mt. Emei, China [J]. Primates, 34(4): 431-444.

Zhao QK, Deng ZY. 1988. Macaca thibetana at Mt. Emei, China. II. Birth seasonality [J]. Am J Primatol, 16(3): 261-268. 